\title{
An unusual cause of distal duodenal bleeding
}

\author{
Jen-Wei Chou ${ }^{1,2,3,4}$, Ken-Sheng Cheng ${ }^{1,2}$, Shih-Chieh Chuang ${ }^{2}$ \\ ${ }^{1}$ School of Medicine, China Medical University, ${ }^{2}$ Division of Gastroenterology and Hepatology, Department of Internal Medicine, China Medical \\ University Hospital, Taichung, Taiwan, ROC.; ${ }^{3}$ Taiwan Society of Inflammatory Bowel Disease (TSIBD); ${ }^{4}$ Taiwan Association for the Study of \\ Small Intestinal Diseases (TASSID)
}
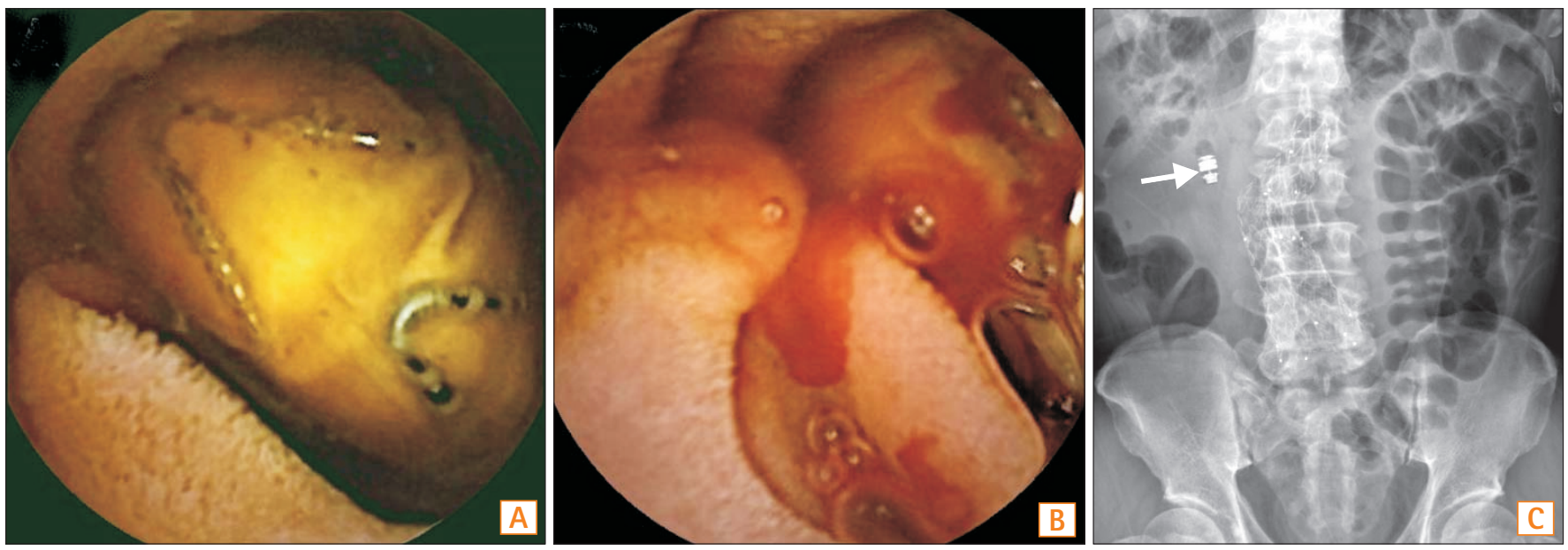

Question: A 63-year-old man was admitted to our hospital because of intermittent fever and anemia for 2 months. He was diagnosed as having abdominal aortic aneurysm rupture with an initial presentation of abdominal pain 4 months previously. He underwent emergency endovascular abdominal aortic aneurysm repair (EVAR) by using an aorto-bifemoral bypass graft. After EVAR, he developed intermittent fever, despite the administration of antibiotics. Blood culture yielded multiple bacterial species. Moreover, anemia with positive occult blood in stool was also found. Esophagogastroduodenoscopy and colonoscopy failed to detect the bleeding point. Thus, mid-gastrointestinal bleeding and a focus of infection in the small intestine were highly suspected by the infectious disease specialist. Capsule endoscopy demonstrated a foreign body with several V-shaped metallic wires coated on its surface in the third portion of the duodenum (Fig. A). Moreover, oozing of blood was identified in the vicinity of the foreign body (Fig. B). Abdominal X-ray showed a retained capsule in the right upper abdomen near a tortured aortic stent (Fig. C, arrow). What is your diagnosis of this foreign body?

\footnotetext{
Received December 14, 2015. Revised December 28, 2015.

Accepted December 30, 2015.

Correspondence to Shih-Chieh Chuang, Division of Gastroenterology and Hepatology, Department of Internal Medicine, China Medical University Hospital, No. 2, Yude Road, North District, Taichung 40447, Taiwan, ROC. Tel: +886-4-22052121 Ext. 2220, Fax: +886-4-22023119, E-mail: codecol80@ gmail.com

Financial support: None. Conflict of interest: None.
} 


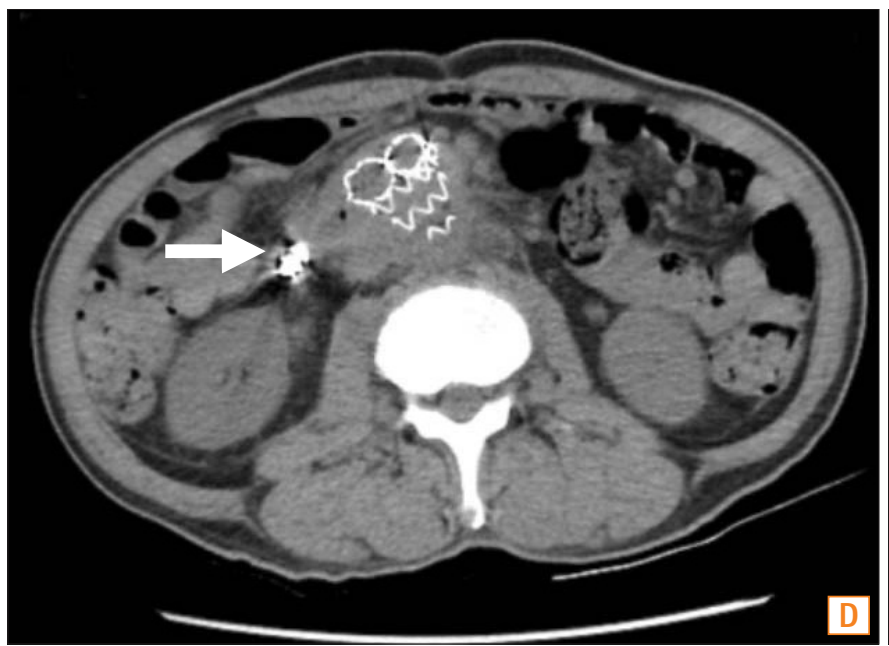

Answer to the Images: Aortic Stent-Induced AortoEnteric Fistula

The findings of capsule endoscopy suggested a migrated aortic stent graft in the distal duodenal lumen with complications of duodenal bleeding and perforation. Non-contrast abdominal CT scan demonstrated a retained capsule near the aortic stent (Fig. D, arrow). Moreover, contrast abdominal CT demonstrated a tortured aortic stent with partial migration into the distal duodenal lumen (Fig. E, arrow). On the basis of these findings, the patient was taken for surgery. An exploratory laparotomy revealed that the aortic stent had been displaced through the distal duodenum, leading to perforation of the wall.

EVAR is an endovascular surgery used to treat abdominal aortic aneurysms, and the long-term survival after this surgery is similar to that following open repair. ${ }^{1}$ Aorto-enteric fistula (AEF) after EVAR is an extremely rare event and most commonly involves the distal portion of the duodenum ( $87 \%$ of cases) and rarely the colon $(2 \%){ }^{2}$ The mechanisms are multi-factorial, the commonest being aortic wall erosions, and other causes may be stent migration, stent infection, or endoleak. ${ }^{3}$ The clinical presentations of AEF are gastrointestinal bleeding, abdominal pain, or fever. Due to the low diagnostic rate on endoscopy, the diagnosis is usually made by abdominal CT, 18-fluorodeoxyglucose PET or surgery. ${ }^{4}$ The gold standard of treatment of AEF is stent removal and extra-anatomic site bypass, but the mortality of open surgi-

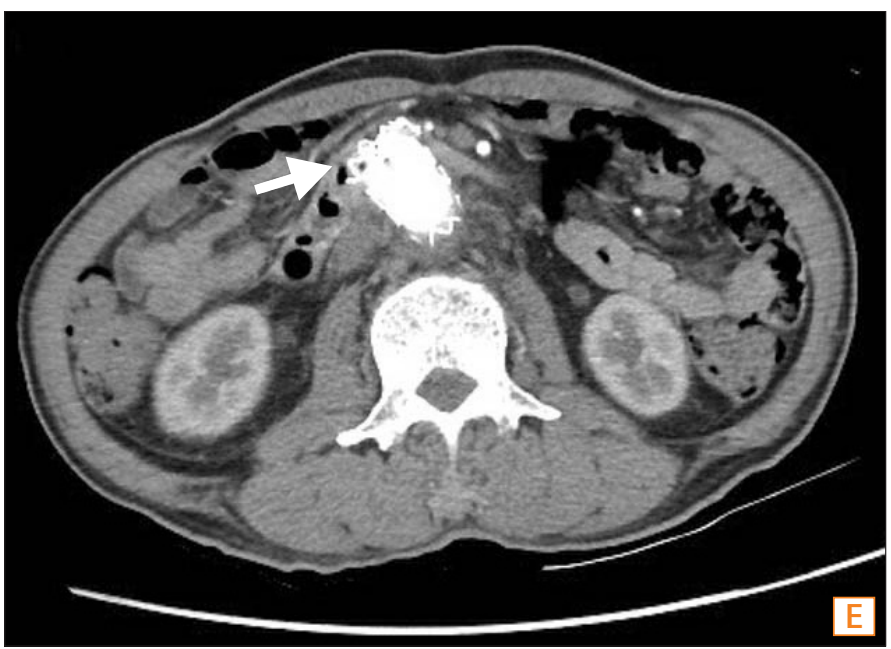

cal repair with an infected stent may be up to $25 \% .{ }^{5}$ Although aortic stent-induced AEF is rare, severe complications occurring after EVAR are difficult to treat. Therefore, duodenal bleeding and perforation due to aortic stent-induced AEF, should be considered as a possible occurrence in patients who underwent EVAR.

\section{REFERENCES}

1. De Bruin JL, Baas AF, Buth J, et al. Long-term outcome of open or endovascular repair of abdominal aortic aneurysm. N Engl J Med 2010;362:1881-1889.

2. Mangiavillano B, Mezzi G, Testoni PA. Usefulness of lower gastrointestinal endoscopy on suspicion of an iatrogenic sigmoid perforation caused by an aorto-bifemoral prosthetic bypass. Endoscopy 2008;40(Suppl 2):E170.

3. Antoniou GA, Koutsias S, Antoniou SA, Georgiakakis A, Lazarides MK, Giannoukas AD. Outcome after endovascular stent graft repair of aortoenteric fistula: a systematic review. J Vasc Surg 2009;49:782-789.

4. Tsunekawa T, Ogino H, Minatoya K, Matsuda H, Sasaki H, Fukuchi K. Masked prosthetic graft to sigmoid colon fistula diagnosed by 18-fluorodeoxyglucose positron emission tomography. Eur J Vasc Endovasc Surg 2007;33:187-189.

5. Cernohorsky P, Reijnen MM, Tielliu IF, van Sterkenburg SM, van den Dungen JJ, Zeebregts CJ. The relevance of aortic endograft prosthetic infection. J Vasc Surg 2011;54:327-333. 